\title{
Capacitive sensors for detecting proximity and response
}

\author{
Steven W. Badelt and Aaron P. Blaisdell \\ University of California, Los Angeles, California
}

\begin{abstract}
We have implemented an inexpensive circuit that indicates when a subject is in close proximity to a specified area of arbitrary shape and size. The circuit can transform an easily created metal surface or a preexisting metal object into an experimental sensor. The subject need not make physical contact with the sensor to activate the mechanism, which enables the detection of subjects through nonconductive materials, such as wood and plastics. The technology is readily adapted to existing experimental setups. Applications of this technology, using pigeons, rats, and mice, demonstrate its flexibility: a nose-poke/peck sensor for response or choice detection; a subject location detector that can be placed on a floor, wall, or ceiling; and a perch that detects when a bird is present. This technology can also be applied to the study of behavior in primates and many other animal species.
\end{abstract}

The goal of any science of behavior is to understand the processes that govern behavior. A prerequisite for this goal is the ability to accurately record and analyze the behavior of the subject under investigation. Although the full richness of an individual's behavior may be most fully captured through visual observation, this method of data acquisition is extremely time consuming, effortful, and prone to error. There has long been an interest in developing automated behavioral-recording systems to overcome the challenges associated with direct observation of subject behavior. Various apparatuses have been constructed in an attempt to reduce, at least in part, the problems of direct observation. A few milestones in the history of these automated behavioral recording systems include Skinner's development of the operant box (Skinner, 1938) and the development of automated video motion-tracking systems (Noldus, 1991).

Modern behavioral-recording systems implement sensors that transduce a behavioral event into an electrical signal. For example, a mechanical lever transfers a specific movement of the manipulandum to an electrical switch contact, transforming the mechanical movement into an electrical signal. An optical sensor produces a change in electrical current when a beam of incident light is broken. These technologies are pervasive in behavioral research because electrical signals can be recorded by control systems that automate behavioral experiments.

There are limitations and drawbacks to the detection of behavioral events with any particular method. Mechanical levers can be used for barpressing and keypecking but may be difficult to adapt to complex detection tasks requiring many discrete choices or to tasks that measure positional behavior (e.g., in a maze or an open field). Such instrumentation may involve costly and complex machining of parts, and mechanical apparatuses are subject to interfer- ence by any debris that may infiltrate the mechanism. Mechanical systems require some form of physical contact by the subject, potentially interfering with the behavior of interest, and moving parts will eventually fail due to wear. Optical sensors avoid the requirement of physical contact and can be used to detect the passage of a subject, nose pokes, or head entry at a hopper. They require separate components for producing and sensing a beam of light, however, which restricts their application to physical configurations that can accommodate this linear arrangement. As with mechanical sensors, debris can accumulate on either the sensor or the light source and prevent proper function. Optical sensors also require proper alignment of the light source and detector, and external light variations can disrupt proper function.

Newer monitoring technologies have minimized some limitations while introducing new ones. The primary advantage of video surveillance is that it records all observable behavior, but such systems are often expensive, difficult to set up, computationally intensive, and space constrained. Video may require post hoc analysis by the investigator for error checking or scoring. The time requirements and accuracy of automated video analysis may preclude its use in experiments that require immediate feedback to subject response. Another alternative is the use of impedance detection circuits to determine when a subject has contacted a surface. These circuits do not impose the same limitations as optical or mechanical measures. This technique, however, requires direct physical contact by the subject, as well as electrical isolation of the circuit (Anger \& Schachtman, 2007).

To our advantage, modern commercially available microchips modularize complex circuits that have previously been custom designed by expert engineers, using

A. P. Blaisdell, blaisdell@psych.ucla.edu 


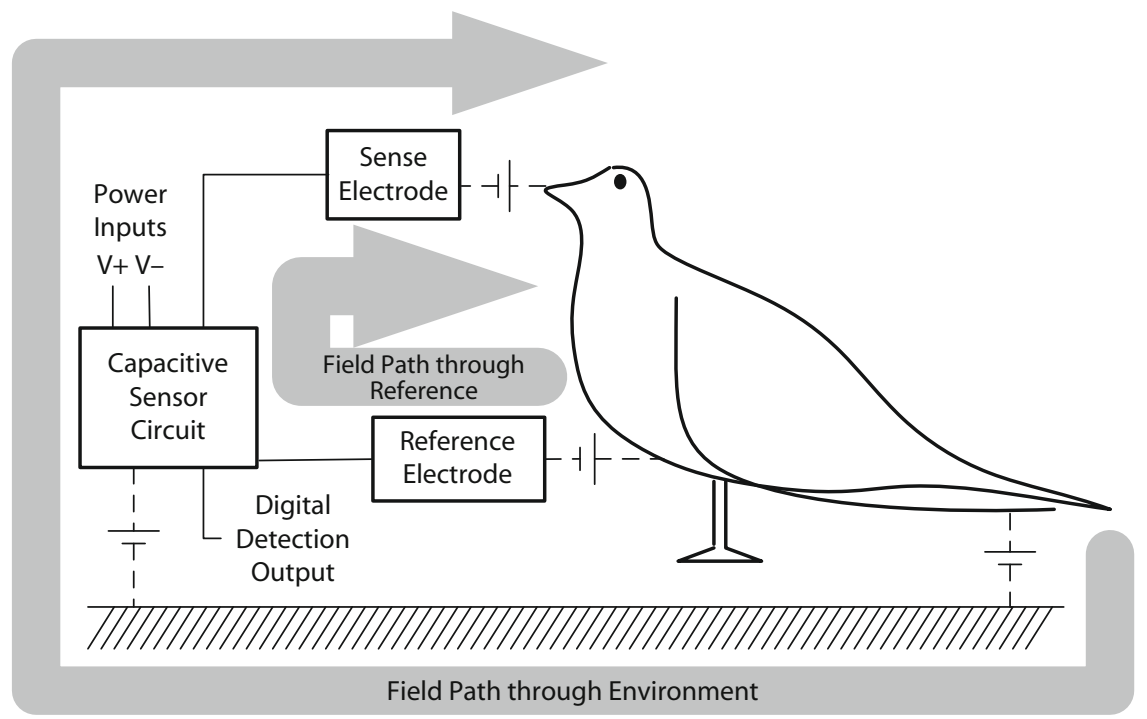

Figure 1. The sensor detects changes in electrical properties generated by interaction from the subject's (in this example, a pigeon) free-space capacitance. If no reference electrode is provided for the detector setup, the capacitive field path returns to the sensor via the environment, represented here by a capacitance to ground, following the large, gray outer loop. In some cases, this path may not be sufficient for proper detection. When an appropriate reference electrode is connected to the circuit, it improves the capacitive return path by creating a second path through the reference electrode, shown as the small, gray inner loop.

many components. Many companies now manufacture integrated circuits (ICs or microchips) that perform advanced functions for sensing the location or proximity of a human finger. When used with a video display device, these microchips can sense a finger placed against a touch screen, an alternative to using infrared technology. Similarly, users of Apple iPod devices are familiar with the dial control that reacts to the movement of a finger across its surface. A major advantage of these electronic sensors is that they do not contain moving parts, which can be costly to fabricate and are prone to wear. These sensing circuits are called proximity sensors.

Because recent microchip technology now integrates the complex parts of proximity circuits into a single component, a behaviorist can produce new experimental detectors with minimal effort and only a few components. We demonstrate a method of behavior detection in which a circuit that electrically detects a subject's responses or proximity to a visually hidden sensor is used. This capacitive proximity sensor may be applied in a variety of situations, and it avoids many of the limitations of present technologies (although we will discuss some of its own limitations at the end of the Method section).

\section{METHOD}

In contrast to mechanical, optical, and electrical-resistive measures, the proximity sensor used in our lab operates by detecting small perturbations in capacitance that are generated by a subject. Capacitance is a measure of electrical charge stored at a given potential. A device that stores a charge in this manner is called a capacitor, and the most basic example is the result when two metal surfaces are placed parallel to one another. The imbalanced placement of electrons, with more on one metal surface than on the other, results in a voltage potential difference between the two surfaces. Although engineers may optimize the parameters of a capacitor so that it will function in one's computer, all physical objects, including experimental subjects, present a capacitance that exists between them and their environment. This is termed the free-space capacitance.

The sensor we have implemented includes a circuit that can detect changes in capacitance, as measured with a sensing electrode. As a subject approaches the sensing electrode, the subject's free-space capacitance changes the total effective capacitance measured between a sensing electrode and the circuit's electrical reference. The sensor circuit measures the capacitance and signals detection upon the event of a significant change (Figure 1). It is not necessary for a subject to directly contact any component of the electrical system for the subject to trigger detection. The impact of a subject on the sensing electrode decreases with increasing distance, and so the sensor's effective range is typically $1 \mathrm{~cm}$ or less from the surface of the sensing electrode.

We have successfully implemented a peck/nose-poke sensor (Figure 2), a location detector (Figure 3), and a perch indicator (Figure 4), using capacitive sensing. Each sensor includes a small sensing circuit, a sensing electrode, and a reference electrode. When a subject is within close proximity to the sensing electrode, the circuit produces a voltage pulse on its electrical output. The sensor has five external connections for function: (1) a positive voltage source $(\mathrm{V}+)$; (2) the voltage source reference $(\mathrm{V}-$, often labeled as COM, $0 \mathrm{~V}$, or reference, and often mistakenly referred to as ground); (3) a digital output signaling whether a subject is detected; (4) an electrical connection to one or 
Top View

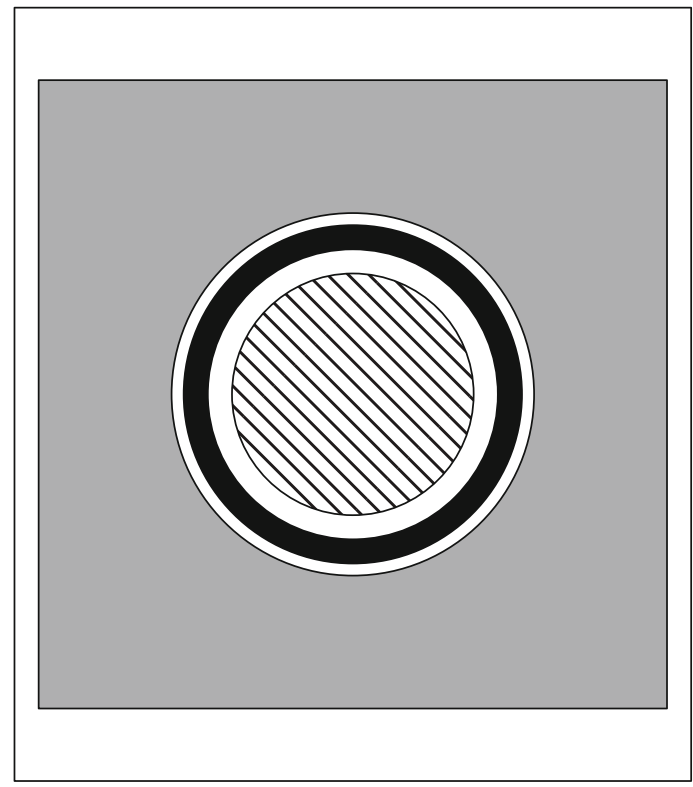

Side View

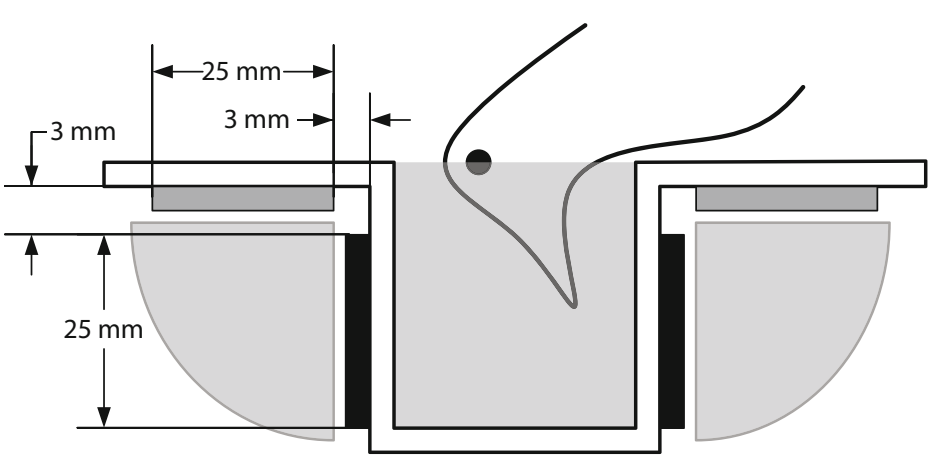

Sensing electrode

Reference electrode

Sensing field

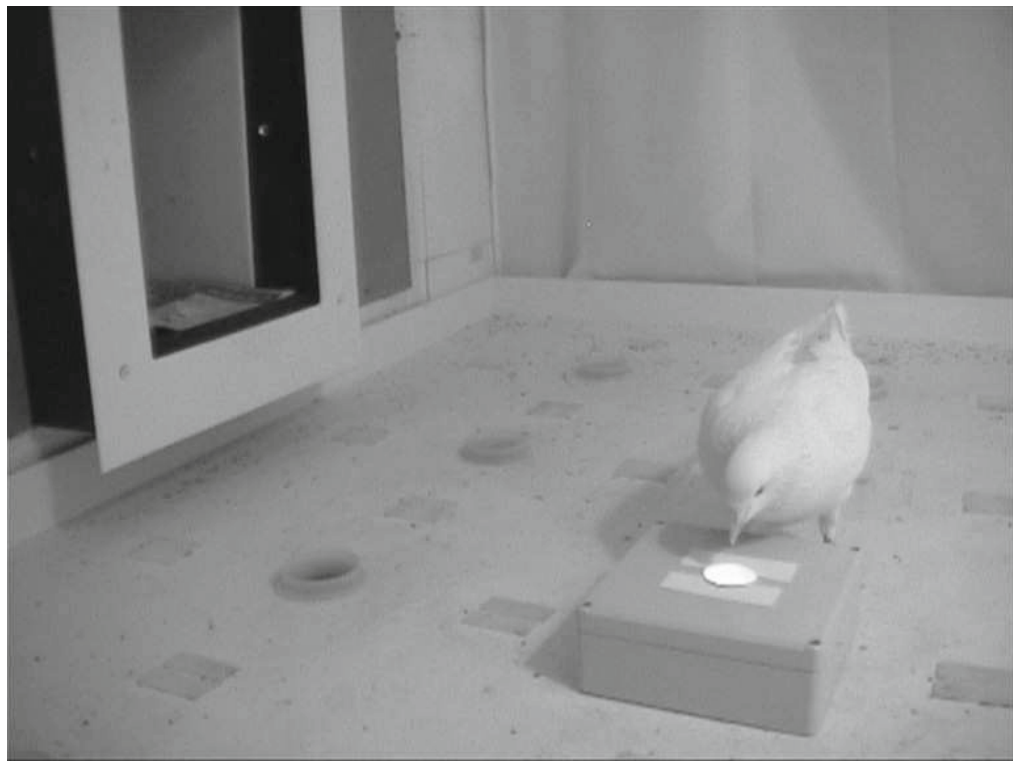

Figure 2. The diagram shows the electrode arrangement for the capacitive proximity detection of pigeons (Leising, Garlick, Parenteau, \& Blaisdell, 2008) performing a peck and of rats performing a nose poke. Due to the electrical properties of a pigeon's beak and the speed of the stereotyped peck, a planar electrode did not robustly detect activity. The recessed position of the sensing electrode (in black) allows a larger portion of the pigeon's head to interrupt the sensing field (hashed lines). The flat panel into which the cup (in white) is mounted is from an ABS plastic enclosure. The structure of the cup is manufactured with inexpensive PVC components readily available at hardware stores. Here, the reference electrode (in gray) surrounds the cup to prevent inappropriate detections when the subject approaches the lip of the cup from the panel surface. The reference electrode also shields the wiring below the panel (not shown) from any field interaction with the subject. The sensing field indicated on the cup-side of the panel indicates that the circuit will also detect an experimenter or subject approaching from the back of the panel. This particular configuration responds to rat nose poking and pigeon beak entry (pecking). The photo shows a pigeon interacting with the device.

more proximity-sensing electrode(s); and (5) an electrical connection to one or more reference electrode(s).

The capacitive coupling of the entire sensor circuit with its environment is often sufficient to operate the sensor without using a reference electrode. We highly recommend, however, that experimenters include a connection to a reference electrode that is paired with the sensing electrode. Although the reference electrode is not always required for the function of the circuit, a properly placed reference electrode will improve detection sensitivity and specificity. 
Top View

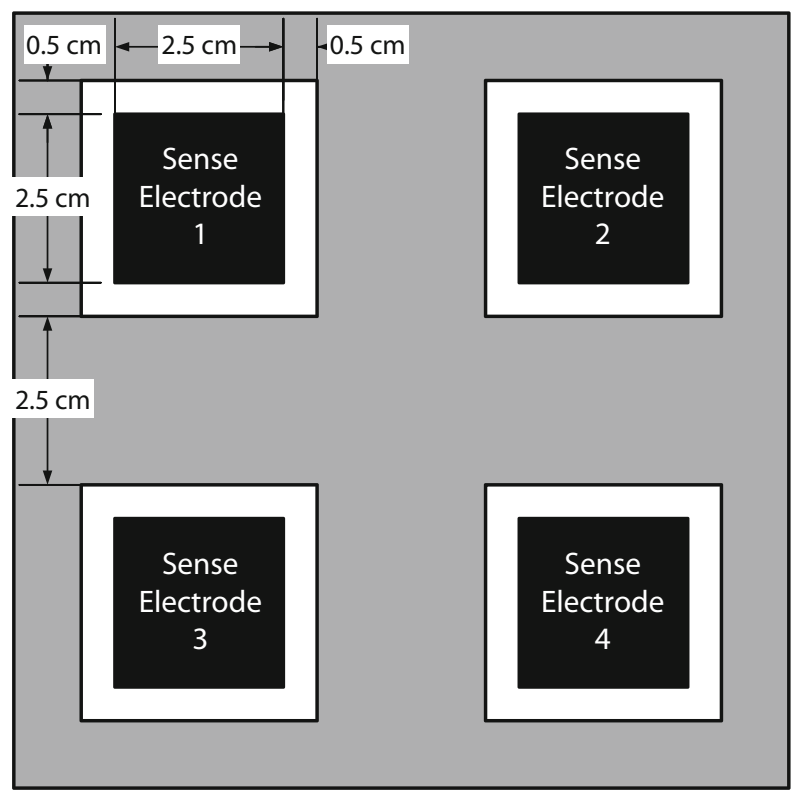

Side View

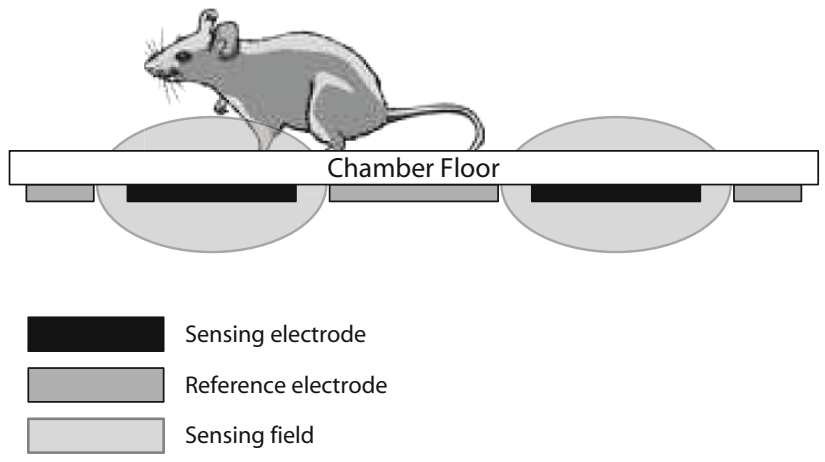

Figure 3. Multiple sensing electrodes (in black) can be situated within a common reference electrode (in gray) plane to individually detect the presence of a subject (in this example, a rat) located at one of many positions along a wall or floor. The geometry of the sensing electrodes and the corresponding gap between each sensing electrode and the reference electrode can be modified according to the subject size and axis of desired localization. For instance, the sensing electrode size optimized for a rat may be larger than that for a mouse. Also, if the experimenter needs to determine the location of the subject in only one dimension along an alley maze, the sensing electrodes can be elongated across the width of the alley and placed in a column along the alley length. We have successfully tested this particular arrangement with mice and rats.

The sensing circuit (Figure 5) incorporates a touch control IC developed by Quantum Research Group (Hamble, Southampton, UK; www.qprox.com). Although originally intended for human interaction, we have demonstrated that these chips can be used for detection and response recording in animal behavior experiments (Leising, Garlick, Parenteau, \& Blaisdell, 2008). Nevertheless, it is important to keep in mind that these products are designed to sense the intentional digit press of a human, not the inadvertent behaviors of smaller subjects. To overcome this limitation, we use the QProx model number QT113H Charge-Transfer Touch Sensor IC, which is designed by the manufacturer for a high level of sensitivity. This chip can be used for a variety of applications, but it must be set up appropriately for each. Our sensor circuit adapts the input and output signals for connection to typical behavior research equipment, including systems from Med Associates Inc. (St. Albans, VT) and Coulbourn Instruments (Allentown, PA).

The input voltage terminals for the sensor circuit are designed to accommodate typical behavioral measurement systems. The sensing microchip uses less than a milliamp of current from a voltage source between 3 and $5 \mathrm{~V}$, although common behavior test systems operate on supply voltages at or exceeding $12 \mathrm{~V}$. The circuit design includes an inexpensive solution for adaptation to a wide range of power supply voltages. Resistor $\mathrm{R}_{\text {supply }}$ (see Figure 5) should be chosen according to the input voltage as computed by the following equation:

$$
\mathrm{R}_{\text {supply }}=\left(\mathrm{V}_{+ \text {input }}-\mathrm{V}_{\text {-input }}-3.3\right) * 200 \text {. }
$$

Resistor values are generally available in fixed increments, so the next value larger than that calculated should be chosen. For instance, systems running on $28 \mathrm{~V}$ (e.g., Med Associates products) should specify $\mathrm{R}_{\text {supply }}=$ $4.99 \mathrm{~K} \Omega$.

The output of the sensing circuit is referenced to the connected power supply rails. When no subject is detected, the output signal is pulled to the supplied V+ power supply voltage. Hence, if the power supply provides $\mathrm{V}+=12 \mathrm{~V}$, the output with no subject present is $12 \mathrm{~V}$, and if the power supply provides $\mathrm{V}+=28 \mathrm{~V}$, the output is $28 \mathrm{~V}$ when no detection is made. When a subject is within the detection range of the electrode, the detection output signal is pulled to the reference signal. In either case, the output is pulled to approximately $0 \mathrm{~V}$ (less than $0.5 \mathrm{~V}$ ) when a subject is detected by the sensor. $R_{\text {pull-up }}$ should be specified according to the equation:

$$
\mathrm{R}_{\text {pull-up }}=\left(\mathrm{V}_{+ \text {input }}-\mathrm{V}_{\text {-input }}\right) * 100 \text {. }
$$

For example, $R_{\text {pull-up }}=2.49 \mathrm{~K} \Omega$ is appropriate for $24-\mathrm{V}$ systems. Some behavior systems, such as those presently produced by Med Associates, already include a pull-up resistor on the detection signal line. For these, the resistor $\mathrm{R}_{\text {pull-up }}$ can be omitted. The circuit can produce short pulse widths on the order of $5 \mathrm{msec}$ in response to fast motion, such as stereotypical pigeon pecks. In order to work with recording systems that cannot be triggered with 


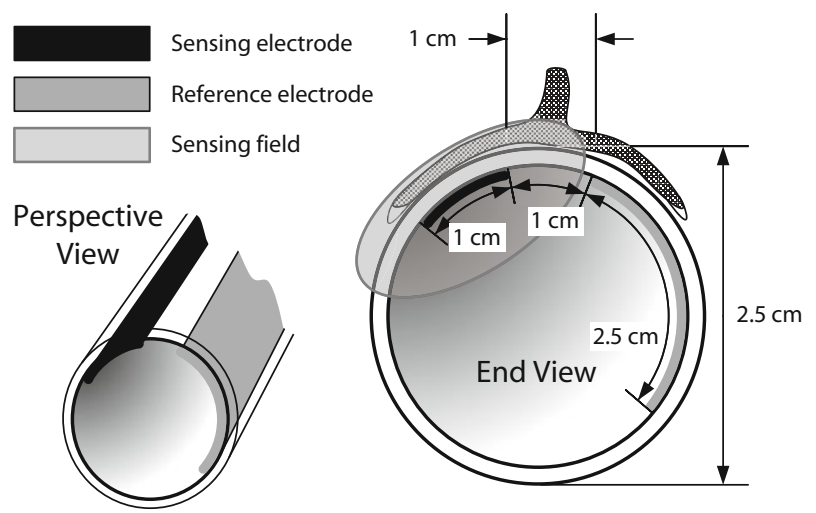

Figure 4. A perch detector is formed from commonly available PVC pipe with copper foil electrodes applied along its interior length. Electrical tape or hot glue is applied to provide a gripping surface.

short pulses, a simple pulse width extender can be added to the output.

The QT113H chip is capable of performing selfinitiated recalibration, but we limit when this function is to be performed. The recalibration function is intended to accommodate environmental drift and changes in electrical noise over time. If recalibration were to occur while a subject is interacting with the sensor, however, proper sensitivity to subject interaction may be compromised. For this reason, the sensor circuit is programmed to self- calibrate only when power is first applied. The calibration will monitor sensor interactions at power-up and, subsequently, classify similar interactions as no detection. Therefore, significant interference with the sensor at power-up may prevent detection afterward, requiring that the system be turned off and on again to force recalibration of the sensor. The experimenter should ensure that the sensor powers up in a known state, with the subject and experimenter out of the sensing range of the circuit's electrodes. In the absence of such an error, we have not found it necessary to force recalibration of the sensor during indoor applications. Nevertheless, the recalibration function may be helpful when environmental conditions change over time, such as with extreme changes of humidity during outdoor applications.

The range of detection can be visualized as a sensing field projected a short distance out from the sensing electrode. This field represents the volume in which a subject will contribute a change in capacitance that is sufficient to trigger a change in the detection output signal. The sensing field size and shape can be modified for individual applications, as indicated by the hashed lines in Figures $2-4$. The effective sense field of the peck sensor is a cylindrical volume (Figure 2), and the location detector consists of multiple square sensing fields confined close to a planar surface (Figure 3). Factors that impact the size and shape of each sense field include the electrical properties of the surrounding materials, the size and shape of the electrode, and the distance between the sensing electrode and the reference electrode.

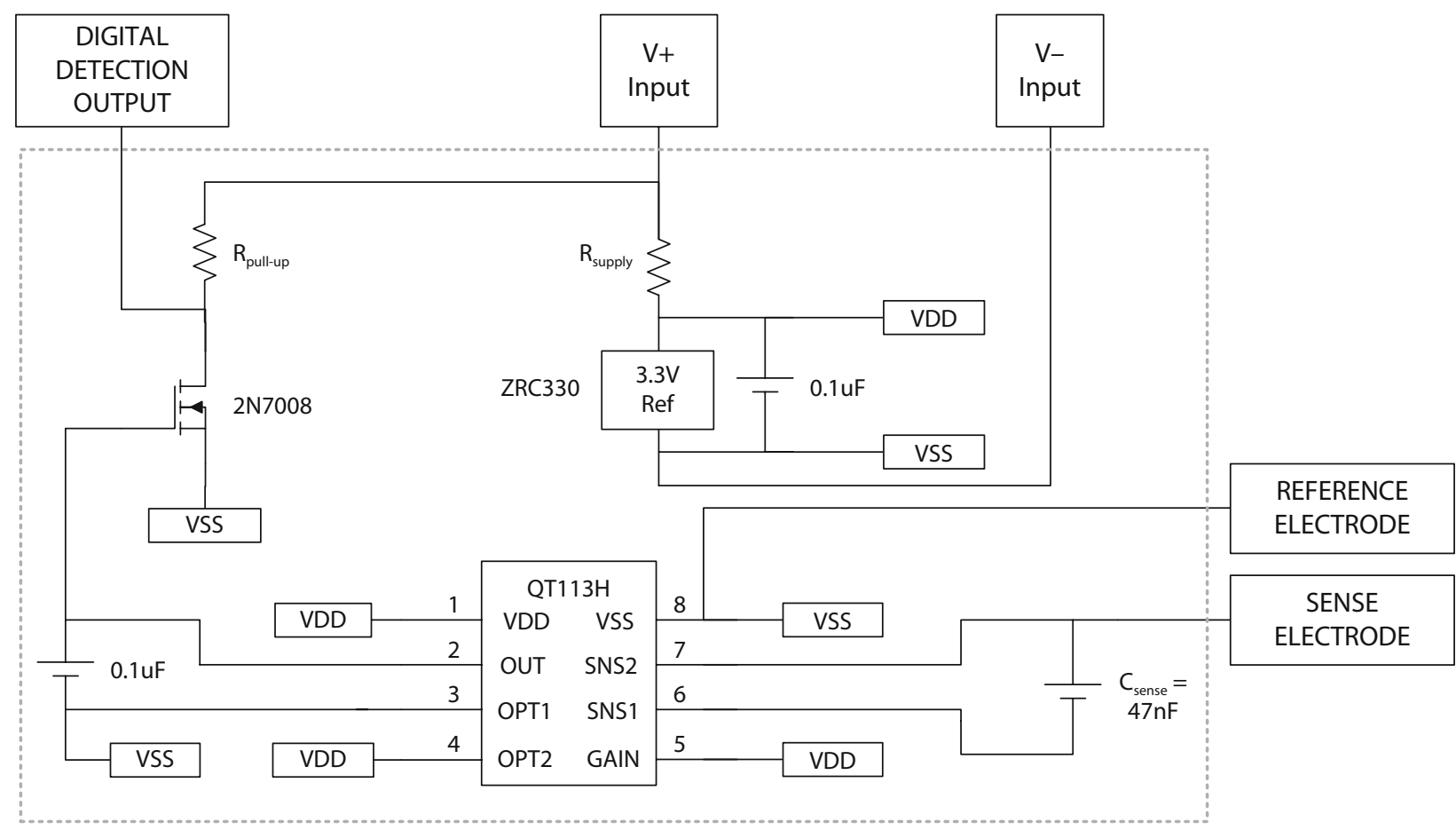

Figure 5. The sensor circuit schematic details the electrical connectivity between eight electrical components. It is sufficient for someone with a basic understanding of electronics to replicate our design. The total cost of parts for this sensor and most electrode configurations is under $\$ \mathbf{1 0}$. If necessary, the circuit sensitivity (i.e., sensing range) can be increased by increasing the value of $\mathrm{C}_{\text {sense }}$, at a cost of slower detection time. Novice circuit builders should note that VDD and VSS designations in the circuit diagram should be electrically connected to all other points with the same labels. 
A

\section{Peck/Poke Sensor}

Observer Reports

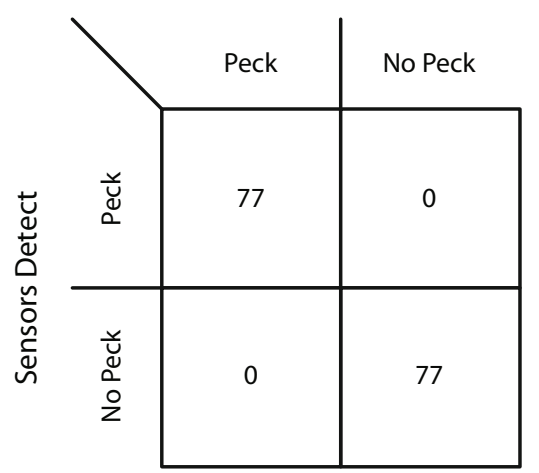

Location Sensor

Observer Reports

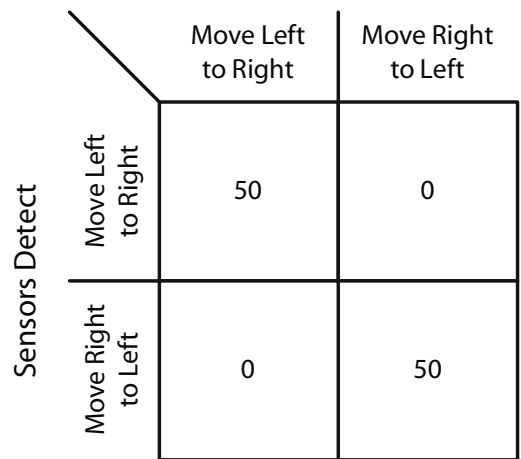

Perch Sensor

Observer Reports

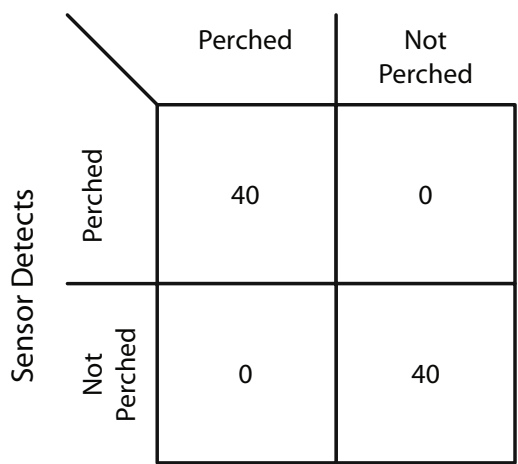

B
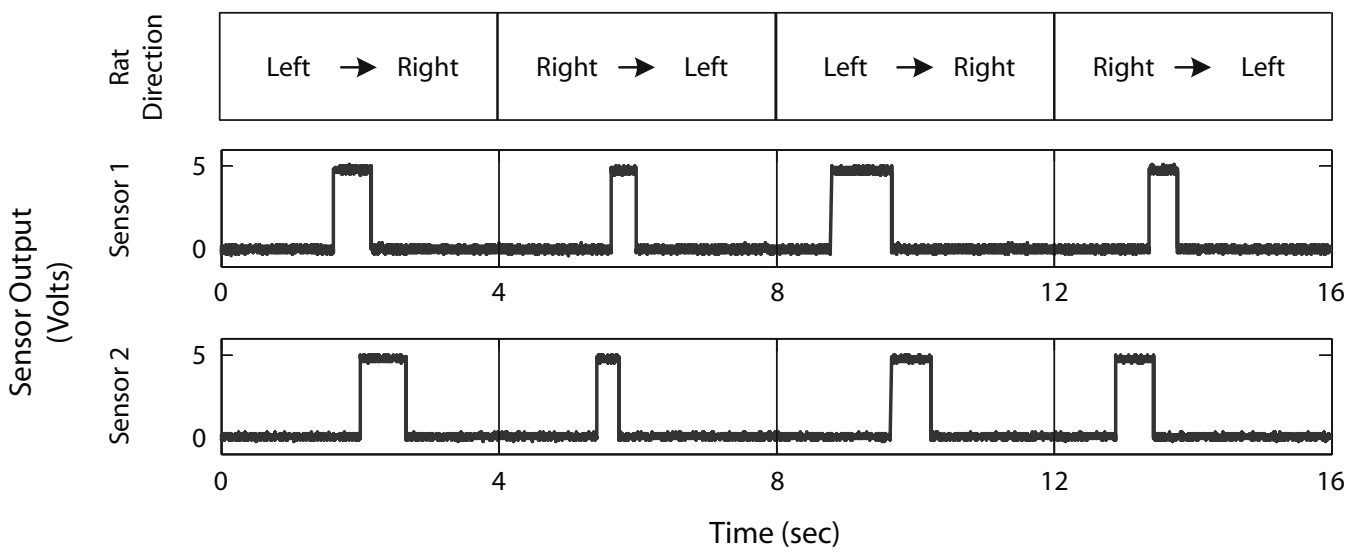

Figure 6. (A) Confusion tables for each of the tests performed on the three sensor installations shown in Figures 2, 3, and 4, respectively. Each demonstrates $100 \%$ accuracy across a modest number of trials while detecting the proximity of a subject as described in the text. (B) Output from two sensors placed on the Plexiglas wall in an alley maze during four concatenated 4-sec segments from individual trials with the same rat. As the rat passed the sensor array, alternating sequence orders demonstrate one sensor's detecting the subject before the other sensor. When each sensor detects the proximity of the rat, its output changes from low $(0 \mathrm{~V})$ to high $(5 \mathrm{~V})$, with the logic high voltage $(5 \mathrm{~V})$ determined by the power supplied to the system $(5 \mathrm{~V})$. As the rat passes by the sensors four times, the sensors correctly indicate that the rat has passed the sensor array in one direction, reversed course, and then passed by the sensor array in the opposite direction. Four consecutive passings are shown.

The sensing field projects through most electrical insulators, including Plexiglas (Figure 3), plastics (Figures 2 and 4), and most dry woods. The sensing and reference electrodes can be mounted on the opposite side of a nonconductive barrier so long as the material thickness is not greater than the range of sensing. For instance, the electrodes can be mounted on the exterior of a T-maze wall or floor and still detect the proximity of a subject, as is diagrammed in Figure 3. The ability to conceal the sensing device enables measurements to be made where visual and tactile cues would detract from the experiment. Alternatively, sensing electrodes can be mounted so that they are exposed to the subject, allowing the subject to make direct contact with the electrode. This would often be the case when a preexisting metal object is used as the sensing electrode. For instance, a stainless steel bar of an operant chamber floor could be set up to indicate when a rat has placed its foot on or near that bar.
Most conductive materials are suitable for construction of the electrodes. It is possible to add electrodes to existing setups by mounting them to nonconductive surfaces. We have had satisfactory results when manufacturing sensing and reference electrodes with conductive-adhesive copper tape (1181 Tape, 3M, Austin, TX) when mounted to insulating materials such as plastics. The advantage of this material is that it can be applied to any clean, nonconductive surface, multiple strips can be overlaid to produce larger electrodes, and arbitrary shapes can be cut using scissors. Furthermore, conductive tape allows the experimenter to produce electrodes that conform to curved surfaces. The sense electrode in the peck sensor of Figure 2 is created by looping conductive tape around a plastic cylinder. By this means, it is possible to produce detection fields of various shapes and sizes. Alternatively, the sensing electrode can be formed from a conductive object in a preexisting setup. It is possible to convert an existing metal surface into a sensing 
electrode by wiring the metal object to the sense electrode connection of the circuit. The metal object cannot, however, itself be connected to the system reference or ground.

The circuit is designed to detect the electrical influence of a subject between the sensing electrodes and the system reference. When the reference electrode is between the subject and the sensing electrode, however, it limits the sensing field and prevents the detection of the subject. Thus, by placing all or part of the reference electrode between the subject and the sensing electrode, the sensing field can be shaped for a particular application. With this mechanism, the experimenter can control the effective volume within which a subject will be detected-such as the restriction of the peck sensing field to the volume inside the cup in Figure 2. Without a reference electrode, it was possible for pigeons and rats to trigger detection by making contact with the surface to which the cup was mounted. With the encircling reference electrode, the effective sense field is limited to within the cup itself.

Proper connection between the sensing and reference electrodes and the sensing circuit is critical to the desired performance. The sensing and reference electrodes are intended to form the exclusive field path by which the sensor detects the subject. The wires connecting the circuit to the electrodes, however, form an alternate capacitive path that acts as part of the electrodes. Therefore, the wires leading from the sensor to the electrodes act as part of the sensor. This has three undesirable consequences. First, when wires move toward or away from each other, the capacitance between them changes. If the electrode wires are loose, they may move relative to one another and hinder proper operation of the circuit. Second, long wires connecting the circuit to the electrodes may increase both the total capacitance and the radio frequency noise measured by the circuit - thereby reducing circuit sensitivity. Third, the subject may approach wires routed adjacent to the experimental area and generate an inappropriate detection. Best practices dictate that the experimenter should keep the electrode wires as short as possible and fixed in position and should route them away from the experimental area if they are not shielded by a reference electrode. If longer wires are needed for connection to the recording system, it is more effective to increase the length of the power and output wires. In general, we have not found these principles onerous, because the circuit is physically small enough to mount on, or close to, the electrodes. With appropriate choice components, the circuit can be manufactured on a circuit board of less than $2 \mathrm{~cm}^{2}$.

There are limitations to the size of the sensing electrode and its proximity to the reference electrode. Making the sensing electrode large increases the capacitance between the sensing electrode and the environment. This capacitance can become too large to allow the circuit to detect the smaller free-space contribution from the subject. Therefore, the size of the sensing electrode is limited. We have successfully implemented sensing electrode sizes over $525 \mathrm{~cm}^{2}$ and over $2 \mathrm{~m}$ long to detect the proximity of an adult rat (approximately $300 \mathrm{~g}$ ), but a 3-m-long electrode $\left(>700 \mathrm{~cm}^{2}\right)$ did not work in the same environment. The capacitance between the sensing and the reference electrodes will also theoretically increase as the separation between the two conductors becomes smaller. Nevertheless, we have not run into any practical limitations with electrodes separated by $5 \mathrm{~mm}$ or more. Notably, the size and separation of the electrodes create a combined effect, and so large parallel electrodes may not result in appropriate subject detection with the separation used for much smaller electrodes.

Before choosing any detector technology, it is important first to determine the requirements for the measurement to be made and determine whether the capabilities and limitations of a given sensor technology are appropriate. Our sensor does present limitations that prevent its application in certain circumstances. For example, it is not suitable for operation in aqueous environments. Also, although the response cup sensor shown in Figure 2 detects responses made by rat nose poking or pigeon beak entry, when mounted to a floor, it may also detect inadvertent actions, such as the insertion of a rat's tail or limb. Similar concerns are relevant to proximity detectors that are mounted directly to the floor surface, such as the subject location detector (Figure 3). Our sensor installations have not detected the presence of feathers, hair, pencils, or other dry debris, but the sensor can be triggered by the presence of conductive debris, including water, urine, feces, or any metal object. This limitation can be avoided when the sensors are, instead, mounted to a vertical surface. When objects or debris remain in the sensing field, it is possible to turn power to the sensor off and then back on, which will force a recalibration. The sensing field of the sensor, however, will most likely be different before and after recalibration in this case. Another alternative is to decrease the sensitivity of the sensor with modifications to the circuit, but this simultaneously reduces the sensitivity to activity from both desired and undesired interactions. If necessary, the construction and modification of this circuit should be within the capabilities of engineering staff members.

\section{TESTING}

Appropriate detection of behavior using the capacitive sensor was compared with simultaneous observation by an experimenter who was blind to the device output. Each of the described configurations (Figures 2-4) was tested in conjunction with animal subject interactions that were consistent with the intended usage.

The peck detector was tested using 1 White Carneau pigeon (Columba livia; $404 \mathrm{~g}$ ) that was previously trained to peck into the detector cup in an open-field setting. A video camera was placed along the surface of the device to which the detection cup was mounted, with the lens pointed toward the cup. This allowed us to judge when the head of the pigeon had entered the cup and, thus, when the sensor should or should not be triggered. At the beginning of each trial, the pigeon was given access to the peck detector (lit with white light from within the cup by an array of light-emitting diodes [LEDs]; see Figure 2, bottom panel), and the observer recorded whether the subject had inserted its beak into the cup. When a detection was recorded by the sensor, the LEDs were extinguished, and 
pellets were made available from a food hopper located in a holding cage adjacent to the open-field room (Figure 2, bottom panel). The hopper was located approximately $1.6 \mathrm{~m}$ from the detector cup. Five seconds after food delivery, the LEDs were relit, and the next trial started. Despite the considerable distance between the food hopper and the detector cup, the pigeon almost always returned to the cup prior to the start of the subsequent trial. Performance was calculated at the end of the 27-min session ( 80 trials, with 3 that timed out before the pigeon responded) and classified in the confusion matrix in Figure 6 (leftmost matrix of panel A). No false detections were recorded by the sensor when the subject was not interacting with the device, and no pecks into the sensor cup were missed by the sensor. In earlier versions of the same sensor with a $22-n F$ capacitor used for $\mathrm{C}_{\text {sense, }}$, we did note that 1 peck was missed by the sensor out of approximately 80 . This highlights the need for appropriate setup, testing, and calibration of detector sensitivity for any new installation. The successful sensing circuit used for the peck detector $\left(\mathrm{C}_{\text {sense }}=47 \mathrm{nF}\right)$ did not include the same input (ZRC330, $\mathrm{R}_{\text {supply }}$ ) or output ( $\left.2 \mathrm{~N} 7008, \mathrm{R}_{\text {pull-up }}\right)$ conditioning components as the circuit described in Figure 5, but the identical setup for all other portions of the circuit performed as specified.

The location detector was manufactured with two $7.6 \times$ $2.5 \mathrm{~cm}$ sensors arranged $98 \mathrm{~cm}$ apart parallel along the longer dimension. Each sensing electrode was surrounded by a reference electrode at a distance of $0.5 \mathrm{~cm}$. This is an arrangement similar to that displayed in Figure 3, with Sensor Electrode 1, Sensor Electrode 2, and the surrounding reference electrode placed with modified dimensions. This device was placed with the sensors mounted to the wall of a straight alley maze, halfway between the two ends. In this arrangement, the rat must pass by each electrode in sequence, triggering one sensor before the other as determined by the direction of travel. In order to ensure that the rat passed close to the sensors, the width of the alley was restricted to 5 or $6 \mathrm{~cm}$. Two Long-Evans rats (Rattus norvegicus; $340 \mathrm{~g}$ each) were placed in the alley during separate sessions. Each time a rat traveled from one end of the alley across the center, the direction of travel was recorded by an observer while timestamps were recorded when each sensor changed state. One of the subjects was observed to repeatedly walk closer to the wall opposite the sensor array, and one of the sensors did not sense this subject on 1 trial when the passage was $6 \mathrm{~cm}$ wide. This highlights the need to test sensor arrangements in novel detection schemes. No missed detections occurred during 40 repeated trials with the passage narrowed to $5 \mathrm{~cm}$. One trial had to be repeated due to attentional deficits on the part of the observer, which highlights the advantages of a well-calibrated detection apparatus. The direction of travel for each rat during 100 center crossings is reported in panel B of Figure 6. No misdetections were observed when either rat was situated away from the sensors (middle matrix of panel A, Figure 6).

The perch detector was tested using 1 White Carneau pigeon (416 g) and 1 Racing Homing pigeon (414 g). A grain cup was placed on one side of the perch to encourage perching. One experimenter repeatedly placed a pigeon on the perch and then removed it from the perch. A second experimenter recorded the output of the perch sensor each time the subject was either on or off the perch. Forty trials were conducted for each pigeon. As in the previous tests, a confusion matrix was generated to summarize the performance of the device (Figure 6, right most matrix of panel A).

\section{CONCLUSION}

We have successfully applied capacitive proximity detection technology to the recording of the behavior of pigeons, rats, and mice (Badelt, Parenteau, Leising, \& Blaisdell, 2006). When appropriate to the experimental demands, these sensors provide a behavior measurement solution with advantages over other methods. The parts for a circuit and electrode arrangement are considerably easier to set up and less expensive than machining for a mechanical detector. The sensor is also considerably cheaper than video surveillance for location or activity detection tasks. It also does not impose any line-of-site requirements that would restrict placement of wiring, hiding places, or other objects while using video systems. Although the cost is similar to that of parts for infrared beam sensors, it avoids the issues of optical interference from debris or external light sources and does not restrict detection to a linear beam. Instead, the flexible arrangement of electrodes can create an arbitrarily shaped volume (determined by arrangement of sensor and reference electrodes) within which the subject is detected. Unlike most other technologies, this sensor can be entirely concealed from view and does not require physical contact with the subject. These characteristics allow us to automate discrimination and response measurements across a variety of species.

The ease and cost of construction for customized detection setups prevents the experimenter from being limited to lab equipment that is already available (and in many cases, suboptimal) and from resorting to observational data when no suitable equipment is available for the species under investigation. When this technology is implemented, preexisting experimental setups made from nonconductive materials do not require drilling or other mechanical modification. This has allowed us to quickly remove and replace electrodes for setup and fine-tuning of the desired field of detection. The power requirements of the QT113H microchip are low enough that we also use it in batterypowered applications. This allows implementation of wireless sensors that can be placed in natural habitats and flexible placement of devices in the lab without the need for cumbersome wiring. Over the past year, this sensor has performed reliably in a number of experiments within our lab (Leising et al., 2008). It offers major advantages over other behavior detection mechanisms in some situations.

The applications of this detection circuit are not limited to the electrode arrangements or applications we have documented. As was demonstrated by our test procedure, the location detection circuit of Figure 3 can be placed across small passages to record the direction of movement across a line, as in shuttling experiments (e.g., Isaacson, Douglas, \& Moore, 1961; Randall \& Riccio, 1969). If this 
same grid is placed on the ceiling of an enclosure, rearing by rats could be detected, provided that the ceiling was low enough. Electrodes could be placed at the top of cylinders to detect investigation of response locations in pole tasks (Brown \& Terrinoni, 1996). Similarly, electrodes could be placed in holes in tree branches to detect birds in the process of storing and retrieving seeds (Clayton \& Dickinson, 1998; Shettleworth \& Krebs, 1982). These devices are also well suited for replacement of damage-prone mechanical response devices for primates, cats, and dogs and can function while mounted behind surfaces that are easy to clean. They also enable the detection of behaviors in hermetic environments without the need for interrupting the seals on existing systems, which may be particularly useful for behavioral assessment of subjects exposed to biohazardous materials.

Use of these devices can also introduce a certain convenience factor into experiments that reduces disruption to subjects. Mounting the sense electrodes and circuit to a small piece of Plexiglas allows for flexible placement of the response detector on a day-to-day basis. Such sensors can be mounted on the sides of plastic home cages to train responses without the need for an expensive operant chamber. This would work particularly well for home cages that stack within each other-such as those often used in the study of home cage activity in transgenic mice-because the home cage could simply be nested into the instrumented cage below while dispensing food or water into the cage.

The described sensor circuit is designed for a discrete detection made by a single electrode. It is not, therefore, possible to measure the continuous distance from a subject with this device. Other capacitive sensing microchips, however, are available to detect interactions with multiple electrodes simultaneously. These have human applications for the detection in discrete locations (e.g., numeric keypads) or along a continuous linear scale (e.g., touchless slider controls). Using these devices, circuit and electrode designs tailored to behavior research may extend the abilities of these sensors to continuous scales of measure and finer measures of subject location, using multiple electrodes.

\section{AUTHOR NOTE}

Support for this research was provided by NIH Grant MH066855 (to A.P.B.). We thank Dennis Garlick, Kenneth Leising, Hwee Cheei Lim, and Mike Parenteau for assistance with data collection and support. Thanks also go to Tom Beckers, Dennis Garlick, Kenneth Leising, W. David Stahlman, and Jared Wong for comments on a preliminary draft of this article. Thanks to Jared Wong for pigeon handling. This research was conducted following the relevant ethics guidelines for research with animals and was approved by UCLA's institutional IACUC. Correspondence and requests for reprints should be addressed to A. P. Blaisdell, Department of Psychology, University of California, 1285 Franz Hall, Box 951563, Los Angeles, CA 90095-1563 (e-mail: blaisdell@psych.ucla.edu).

\section{REFERENCES}

Anger, D., \& Schachtman, T. R. (2007). A sensitive, reliable inexpensive touch detector. Journal of the Experimental Analysis of Behavior, 87, 151-153.

Badelt, S. W., Parenteau, M. A., Leising, K. J., \& Blaisdell, A. P. (2006, March). ARENA: Automated remote environmental navigation apparatus. Paper presented at the Annual International Conference on Comparative Cognition, Melbourne, FL. Available at pigeonrat.psych .ucla.edu/Presentations/Badelt-CO3-2006-ARENA.ppt.

Brown, M. F., \& Terrinoni, M. (1996). Control of choice by the spatial configuration of goals. Journal of Experimental Psychology: Animal Behavior Processes, 22, 438-446.

Clayton, N. S., \& Dickinson, A. (1998). Episodic-like memory during cache recovery by scrub jays. Nature, 395, 272-274.

IsaACSON, R. L., Douglas, R. J., \& Moore, R. Y. (1961). The effect of radical hippocampal ablation on acquisition of avoidance response. Journal of Comparative \& Physiological Psychology, 54, 625-628.

Leising, K. J., Garlick, D., Parenteau, M., \& Blaisdell, A. P. (2008). Behavioral research in pigeons with ARENA: An automated remote environmental navigation apparatus. Manuscript in preparation.

Noldus, L. P. J. J. (1991). The Observer: A software system for collection and analysis of observational data. Behavior Research Methods, Instruments, \& Computers, 23, 415-429.

Randall, P. K., \& Riccio, D. C. (1969). Fear and punishment as determinants of passive-avoidance responding. Journal of Comparative \& Physiological Psychology, 69, 550-553.

Shettleworth, S. J., \& Krebs, J. R. (1982). How marsh tits find their hoards: The roles of site preference and spatial memory. Journal of Experimental Psychology: Animal Behavior Processes, 8, 354-375.

SkINNER, B. F. (1938). Behavior of organisms. New York: Appleton.

(Manuscript received August 13, 2007; revision accepted for publication December 21, 2007.) 